\title{
On super-resolution in astronomical imaging
}

\author{
K. G. Puschmann and F. Kneer
}

\author{
Universitäts-Sternwarte, Geismarlandstr. 11, 37083 Göttingen, Germany \\ e-mail: kgp@uni-sw.gwdg.de
}

Received 12 October 2004 / Accepted 27 January 2005

\begin{abstract}
Our work is an attempt to fulfil one of the aims of astronomical imaging, that is, to obtain information at high angular resolution. Stars as point sources can be represented by Dirac $\delta$-functions whose Fourier transforms contain information about the position and the (integrated) intensity at all angular frequencies. Thus, we can deconvolve unresolved images of star fields even at angular distances smaller than the diffraction limit of the telescope with which the observations are performed. We give an example of reconstruction of the image of two stars with an angular separation of a factor 2.5 less than $\alpha_{\mathrm{Ra}}=1.22 \times \lambda / D$. However, we find that super-resolution is feasible only for point sources. For extended objects the information about intensity fluctuations at angular frequencies $u>u_{\max }=D / \lambda=1 / \alpha_{\min }$ is irretrievably lost. We discuss the impossibility of superresolution for the Sun using a numerically simulated image of solar granulation. However, one can enhance the contrast of solar images, though without increasing angular resolution beyond the diffraction limit.
\end{abstract}

Key words. techniques: high angular resolution - techniques: image processing

\section{Introduction}

Large telescopes for astronomical observations have been built to collect as much light as possible and to achieve high angular resolution. For circular apertures the diffraction limit of angular resolution is $\alpha_{\min }=D / \lambda$, where $D$ is the telescope aperture and $\lambda$ the wavelength at which the observations are performed. The modulation transfer function $(M T F)$ of the telescope is zero at wave-numbers $u \geq u_{\max }=1 / \alpha_{\min }$, which means that the angular diffraction limit $\alpha_{\min }$ is smaller than the commonly used definition by Rayleigh $\alpha_{\mathrm{Ra}} \approx 1.22 \times \alpha_{\min }$, which is located at the first zero of the point spread function ( $p s f)$ of the telescope.

It is widely believed that information about angular scales $\alpha<\alpha_{\min }$ is not attainable from observations with $D$ and $\lambda$. Biraud (1969) and Lucy (1992a) used the analytic continuation argument to point out that this limit may be overcome for band-limited images. For low-noise data with enough information at angular wave-numbers $u<u_{\max }=1 / \alpha_{\min }$, superresolution can be achieved, i.e. an angular resolution better than the diffraction limit. Many methods for image deconvolution have been developed. Wittmann (1971) proposed "deconvolution by first convolving with the point spread function" to eliminate seeing effects from solar images. Richardson (1972) and Lucy (1974) then suggested an iterative method based on Bayes' theorem for conditional probabilities, which is known as the Richardson-Lucy algorithm. We refer to the review by Lucy (1994a) who also addressed maximum enthalpy methods and offered more references (see also Lucy 1994b).

Substantially improved methods of deconvolution, which avoid the "ringing" effect around the images of point sources, were developed and explained by Lucy (1994c),
Hook \& Lucy (1994), and Lucy \& Walsh (2003). The methods were termed "two-channel" deconvolution since the images from point sources and from the background are treated separately. Pointing out the mandatory need for correct sampling, Magain et al. (1998) introduced a strong prior knowledge about stellar point sources and reconstructed images of stellar fields with angular resolution much better than the diffraction limit and with high positional and photometric accuracy.

Our main interest here is to improve the resolution of solar images. Based on Biraud's (1969) formulation of a deconvolution algorithm, Miura et al. (1999) suggested a method for theoretically achieving super-resolution of solar images two times higher than the telescopic diffraction limit $u_{\max }=2 / \alpha_{\min , \mathrm{Tel}}$. In our opinion, this is not possible for extended, not band-limited objects.

In the following we present our reasoning together with examples. We show why super-resolution is feasible for stellar objects, even for crowded star fields. We continue discussing the deconvolution of solar images and show that their contrast may be increased but not their spatial resolution.

\section{Super-resolution for point sources}

For the mathematical formulations, we shall use the following notation below. Arrays in the image domain, like image $i$ and point spread function $p s f$, will be denoted by lower case letters. In the Fourier domain we use upper case letters, like $I$ for the Fourier transform of $i$ and $M T F$ for the modulation transfer function that is the Fourier transform of the $p s f$. The $p s f$ of a circular, unobstructed, and diffraction-limited telescope 
with aperture $D$ observed at a wavelength $\lambda$ is the Airy function (Born \& Wolf 1970)

$$
p s f_{D}(\alpha)=\frac{1}{\pi}\left[J_{1}\left(\frac{\pi D}{\lambda} \alpha\right) / \alpha\right]^{2},
$$

where $\alpha$ measures the angular distance from the centre of the $p s f$, and $J_{1}$ is the Bessel function of order 1 . The Fourier transform of this $p s f$ is the rotationally symmetric, real function

$$
M T F_{D}(u)=\frac{2}{\pi}\left[\arccos \left(u / u_{\max }\right)-\frac{u}{u_{\max }} \sqrt{1-\left(u / u_{\max }\right)^{2}}\right],
$$

with $u_{\max }=D / \lambda$. For $u>u_{\max }$ the $M T F$ in Eq. (2) is zero.

In the next section we will deal with extended objects, for which super-resolution is not possible, while here we concentrate on point sources. As long as the surfaces of stars are not resolved, stars may be considered as Dirac $\delta$-functions that carry information at all angular frequencies (in the Fourier domain).

An image of a star is the total amount of light $a$ collected by the telescope times the $p s f$ of the observing process shifted to its position in the field of view (FOV)

$i_{\text {star }}=a \times p s f(x-\Delta x, y-\Delta y)$,

with $x$ and $y$ measuring angular coordinates from the centre of the FOV along rectangular axes. Its Fourier transform is given by

$I_{\text {star }}=a \times M T F(u, v) \times \mathrm{e}^{2 \pi j(u \Delta x+v \Delta y)}$.

This shows that the amplitude $a$ and the shifts $(\Delta x, \Delta y)$ are not lost by the limited resolution. They are retained at angular frequencies $u<u_{\max }$.

Images of two stars with equal brightness, $i_{1}=i_{2}$, and located at equal distances from the centre of the FOV at $\Delta x=\Delta$ and $\Delta y=0$ possess the Fourier transform

$I_{1}+I_{2}=\operatorname{MTF}(u, v) \times 2 a \cos (2 \pi u \Delta)$,

which demonstrates that the $M T F$ is in any case modified, whatever cutoff $u_{\max }$ may be. Yet the factor $\cos (2 \pi u \Delta)$ will remain very close to one for all $u<u_{\max }$ in cases where $\Delta$ is small. Thus $I_{1}+I_{2}$ in Eq. (5) may no longer be distinguished from the image of a single star or any other combination of close stars when the noise is not sufficiently low, as discussed by Lucy (1992a,b).

With the a priori knowledge that one deals with an image $i$ of $K$ stars, the merit function - leaving out normalisation by noise and regularisation by a smoothing entropy function - is

$E=\sum_{i=1}^{N}\left[I\left(u_{i}, v_{i}\right)-\operatorname{MTF}\left(u_{i}, v_{i}\right) \sum_{k=1}^{K} a_{k} \mathrm{e}^{2 \pi j\left(u_{i} \Delta x_{k}+v_{i} \Delta y_{k}\right)}\right]^{2}$,

where $N$ is the number of pixels in the observed image. This equation is a simplified version of the error metric by Magain et al. (1998, their Eq. (7)).

An example of super-resolution with artificial data is given in Fig. 1. For the sake of demonstration, we adopt a simple case and use some of the input as prior knowledge for the reconstruction. We position two stars of equal brightness at equal distances, but in opposite directions, from the centre of the FOV
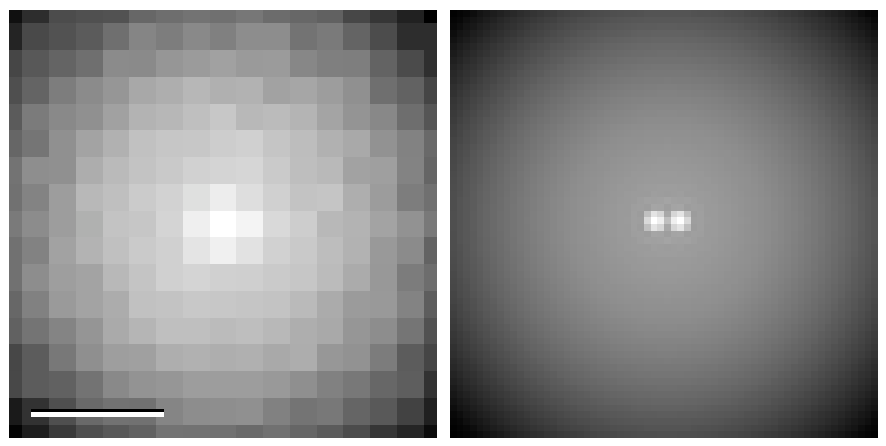

Fig. 1. Super-resolution of a simulated image containing a smooth background and two stars separated by an angular distance a factor 2.5 less than the diffraction limit $\alpha_{\mathrm{Ra}}=1.22 \times \lambda / D$. Gaussian noise is included. Representation of intensities is on a logarithmic scale. Left panel: simulated data; right panel: reconstructed image assuming a telescope aperture a factor 4 larger than for the simulated data. The bar on the left image corresponds to 0.' 5 for observation at $\lambda=500 \mathrm{~nm}$ with a telescope with an unobstructed aperture of $D=50 \mathrm{~cm}$. See the text for more details.

along the horizontal coordinate (cf. Eq. (5)). The distance of the stars is a factor 2.5 smaller than Rayleigh's diffraction limit $\alpha_{\mathrm{Ra}}=1.22 \times \lambda / D$ of the telescope with which the "observations" are performed. It includes smooth background intensity, for which we adopt a Gaussian distribution centred at the centre of the FOV. Also, Gaussian noise is added: a general, positive background noise with constant $\sigma=1 \%$ of the maximum intensity and noise with a $\sigma$ proportional to the square root of the simulated intensities, such that its standard deviation is $3 \%$ of the maximum intensity. The sampling corresponds to 2.5 pixels at an angular distance of the diffraction limit $\alpha_{\mathrm{Ra}}=1.22 \times \lambda / D$.

Given the strong prior knowledge, the only values determined by a least-squares fit to the data of $32 \times 32$ pixels are: the integrated intensity of one star, the distance $\Delta$ from the centre of the FOV, and the maximum of the Gaussian background (assuming its width is known). Background intensity is always retrieved with accuracy better than $1 \%$. The intensities of the stars are recovered within $2 \%-20 \%$, along with the position $\Delta$ within $0.1-0.15$ pixel of the original sampling, depending on the realization of the noise. Obviously, super-resolution is possible with good photometric accuracy and good retrieval of position for point sources like stars. In addition, Fig. 1 does not exhibit any "ringing" from the reconstruction process.

\section{Super-resolution for extended objects}

It is preferable and common practice to define the diffraction limit for extended objects by $\alpha_{\min }=\lambda / D$ or, in Fourier space, by maximum angular frequency $u_{\max }=D / \lambda$, where the $M T F$ of a telescope with aperture $D$ has reached zero. The $M T F$ is thus not an analytic function. Likewise, the Fourier transforms of diffraction-limited images are not analytic functions, but instead identically zero (apart from noise) at frequencies $u>u_{\max }$. In contrast to images from point sources, superresolution is not feasible for extended objects which are not band-limited. The $M T F$ acts as a low-pass filter. Any information at angular frequency $u>u_{\max }$ is lost and there exists no 
possibility of retrieving it. In fact, it is easy to think of objects with a short-wavelength texture that is totally lost when observed with a telescope with too small an aperture. An example would be the object

$i_{\text {texture }}(x)=b \sin ^{2}\left(2 \pi x / \alpha_{\text {texture }}\right)-c$,

with angular wavelength $\alpha_{\text {texture }}>0.5 \times \lambda / D$, where the subtraction of the constant $c=\frac{1}{2} b$ leaves the average intensity of the original image $i_{\text {orig }}$ unchanged. Amplitude $b$ must be small enough to retain the positivity constraint of the total image $i=i_{\text {orig }}+i_{\text {texture }}$. The high-frequency additive term in Eq. (7) has been written in the given form for reasons discussed below, cf. Eqs. (17) and (18). This texture is certainly not realistic and not representative of astronomical objects, below we give examples which do contain power at high angular frequencies. The amplitude and phases at these high frequencies need to be known for correct representation of the underlying object.

Miura et al. (1999) have suggested that it is theoretically possible to increase the angular resolution of a diffractionlimited telescope with aperture $D_{1}$ by a factor of two in the following way. Let $o$ be the object and $i_{1}$ and $i_{2}$ the images of $o$ observed with two different telescopes having unobstructed, circular apertures $D_{1}$ and $D_{2}=2 \times D_{1}$, and the corresponding point spread functions $p s f_{1}$ and $p s f_{2}$, respectively. Images $i_{1}$ and $i_{2}$ are given by

$i_{1}=o \otimes p s f_{1}$,

$i_{2}=o \otimes p s f_{2} \geq 0$.

Since image $i_{2} \geq 0$, it can be represented by

$i_{2}=r^{2}$,

where $r$ is a real function. Transforming into Fourier space yields

$I_{1}=O \cdot M T F_{1}$,

$I_{2}=F\left(r^{2}\right)=R \otimes R=O \cdot M T F_{2}$.

Miura et al. (1999) suggested that the Fourier transform of $r$, denoted by $R$, needs only be known up to $u_{\max , 1}$. Its autoconvolution $R \otimes R$ transports information to $2 \times u_{\max , 1}$. The inverse Fourier transform $F^{-1}(R \otimes R)$ yields the image sought $i_{2}$ with improved resolution. From Eqs. (11) and (12) one obtains

$(R \otimes R) \cdot M T F_{1}-I_{1} \cdot M T F_{2}=0$.

Actually, one may assume a much larger aperture for $D_{2}$. For $D_{2} \rightarrow \infty$ one has $M T F_{2} \rightarrow 1.0, i_{2} \rightarrow o, R \otimes R \rightarrow R^{\prime} \otimes R^{\prime}=O$, and Eq. (13) becomes equivalent to Eq. (11)

$\left(R^{\prime} \otimes R^{\prime}\right) \cdot M T F_{1}-I_{1}=0$.

We have solved the non-linear Eq. (13), or Eq. (14), iteratively for $R=R_{0}+\delta R$ with an approximation $R_{0}$ and a small correction $\delta R$. Neglecting the quadratic term $\delta R \otimes \delta R$ and denoting by $F$ and $F^{-1}$ both the Fourier transformation and its inverse, respectively, one obtains, after some algebra,

$\delta R \approx \frac{1}{2} F\left(F^{-1}\left(\left(I_{1} \cdot M T F_{2}-I_{20} \cdot M T F_{1}\right) / \widehat{M T F}_{1}\right) / r_{0}\right)$.
Here, $I_{20}$ is the approximation of $I_{2}$ and $r_{0}=F^{-1}\left(R_{0}\right)$ the approximation of $r$. Since $M T F_{1}=0$ at $u \geq u_{\max , 1}$ we have replaced it in the denominator on the right hand side of Eq. (15) by a pseudo-modulation transfer function $\widehat{M T F_{1}}$. We found it sufficient to limit it by

$\widehat{M T F_{1}}=M T F_{1}$ for $M T F_{1}>\beta$ and

$\widehat{M T F_{1}}=\beta$ elsewhere

with $\beta=0.001 \ldots 0.01$. At these $\beta$, wavenumber $u$ is close to the maximum wavenumber $u_{\max , 1}$ of the smaller telescope.

We applied the above iterative method to an image from numerical simulations of the solar granulation carried out by the Lindau-Chicago group (Vögler \& Schüssler 2003; Vögler et al. $2005)^{1}$. The image stems from a simulation run without magnetic fields and gives the granulation intensity in the continuum at $500 \mathrm{~nm}$. It has a pixel size of $20.83 \times 20.83 \mathrm{~km}^{2}$ on the Sun (corresponding approximately to $0{ }^{\prime} 029$ ) and is free of noise. It may thus be considered as "ground truth". In the left panel Fig. 2 shows the image when observed with a telescope where $D_{1}=45 \mathrm{~cm}$ corresponding to $\alpha_{\min }=0.23$. The middle panel in Fig. 2 gives the image reconstructed from the left image and the original image is represented in the right panel.

The reconstruction is successful, since the middle image in Fig. 2 is substantially sharper than the "observed" image in the left panel of the same figure. However, reconstruction was only possible to the diffraction limit $\alpha_{\min }=\lambda / D_{1}$, as demonstrated in Fig. 3, where the azimuthally averaged power spectra of the images in Fig. 2 are depicted together with $M T F_{1}^{2}$, but where high powers at $u=0$ resulting from the average intensities are taken out. The power of the reconstructed image follows the power of the original image up to $u=u_{\max }=D_{1} / \lambda$ closely. From there on it drops by two orders of magnitude and then further decreases rapidly. It is found from isolation of this latter part that it contains low-amplitude ringing that is also visible, upon close inspection, in the reconstructed image. The reconstruction acts like a selfconsistent Wiener filter $W$, in this case

$W=\frac{T}{M T F_{1}}$,

with a tapering low-pass function $T$ which brings the filter to zero at high angular frequencies.

The original, "ground-truth" image does not show band limitation. The problem obtaining angular resolution beyond the diffraction limit lies in the infinity of solutions of $R$ in Eqs. (13) or (14). As already mentioned, one may add an artificial high-wavenumber image to the original image, i.e. one may replace $r^{2}$ in Eq. (10) by

$r^{2} \rightarrow(r+z)^{2}=r^{2}+h^{2}-c$

with

$z=-r+\left(r^{2}+h^{2}-c\right)^{1 / 2}$

where the constant $c$ is the average of texture $h^{2}$. Any added image that both contains only fluctuations with sufficiently small amplitude, with sufficiently high wavenumbers, and leaves the average intensity of the observed image unchanged also fulfils

\footnotetext{
${ }^{1}$ We are indebted to Dr. A. Vögler for putting the granulation image from his numerical simulations at our disposal.
} 

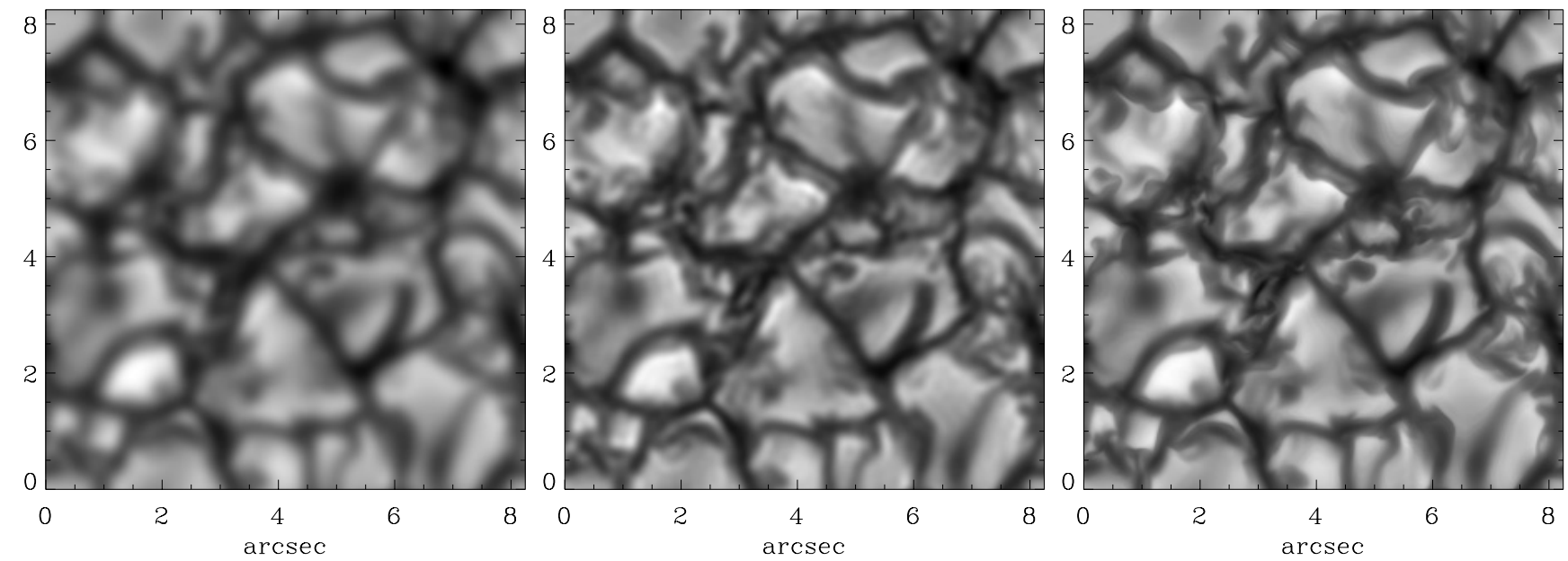

Fig. 2. Reconstruction of an image at $500 \mathrm{~nm}$ obtained from a numerical hydrodynamic simulation of granular convection. Left: image when observed with a diffraction-limited telescope with an unobstructed aperture of $D=45 \mathrm{~cm}$; middle: reconstructed image with the method according to Eqs. (13) to (15); right: original image.

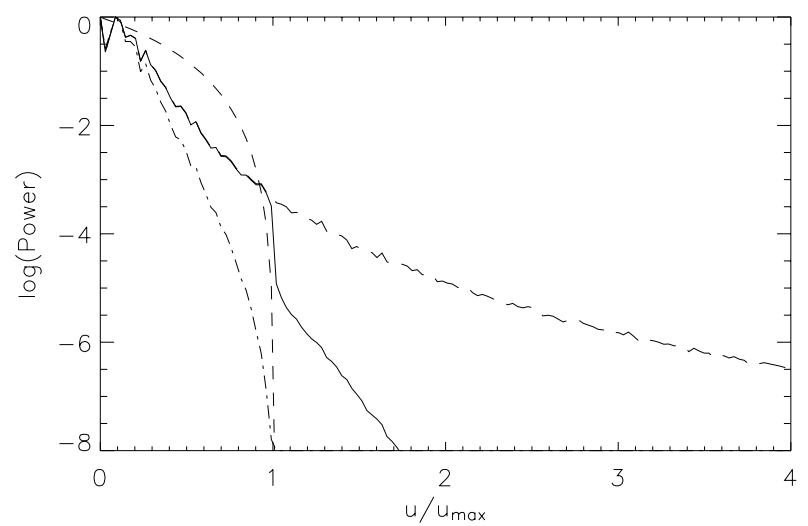

Fig. 3. Azimuthally averaged power spectra from the numerical hydrodynamic simulation. Dash-dotted: power from image observed with a telescope of $D=45 \mathrm{~cm}$; short-dashed: $M T F^{2}$ of diffraction-limited $45 \mathrm{~cm}$ telescope at $500 \mathrm{~nm}$; solid: power of reconstructed image; longdashed: power spectrum of original image at $500 \mathrm{~nm}$.

Eqs. (13) or (14). There exists no a priori knowledge about the very small-scale fluctuations in a solar image, so we have no knowledge about edges, such as those seen in the right image of Fig. 2, or about their sharpness, positions, and lengths. This information, at $u>D_{1} / \lambda$, is lost when observing with too small a telescope.

\section{Contrast enhancement of solar images}

Although we found it impossible to improve spatial resolution beyond the telescopic diffraction limit for images that are not band-limited, it is nevertheless of interest to sharpen these images to enhance the contrast. We give two examples here for sharpening observed solar images, speckle-reconstructed and with very little noise. The reconstructions were performed with the spectral ratio method of von der Lühe (1984) and with the speckle masking method of Weigelt (1977). Also see de Boer \& Kneer (1992) and de Boer (1996).
We used the same iteration method formulated in Eq. (15), which is equivalent to the Wiener filter of Eq. (16), as for the numerical simulation images of Figs. 2 and 3. Some speckle codes do not include the telescopic $M T F$ in the speckle transfer function $(S T F)$. In this case, the sharpening by contrast enhancement with a consistent Wiener filter is a correct procedure for closer approximation to the underlying object. The speckle code applied for the examples below does include the MTF in the $S T F$. Thus, on one hand, fluctuation amplitudes at high wavenumbers may be overemphasised by the sharpening process. On the other hand, the tapering filter, which brings the filter $1 / S T F$ to zero at high wavenumbers and suppresses noise in the speckle code, is often chosen very conservatively such that the amplitude of the intensity fluctuations at high wavenumbers are usually underestimated. Besides, improved visualisation by sharpening, i.e. contrast enhancement, is often desirable when studying solar finestructures.

Figure 4 gives two (sub-)images of the same scenery on the Sun taken in the $G$ band in June 2004 with the Swedish $1 \mathrm{~m}$ Solar Telescope on La Palma and reconstructed with speckle techniques. The sampling was 2.2 pixels across the resolution element. The original, speckle-reconstructed image was interpolated with splines "under tension" to arrive at three pixels within the former two pixels. Cubic interpolation gave results which are not distinguishable from images like those in Fig. 4. The sharpening procedure was then applied, the result of which is shown in the right image of Fig. 4.

The contrast of finestructure has evidently increased compared to the original image. The intensities of the $G$-band bright points have risen from a maximum of 1.6 relative to the mean background intensity to 1.8 , a value compatible with the results from numerical simulations of small-scale magnetic fields by Shelyag et al. (2004) and the resulting $G$-band intensities in their work.

Another interesting result is that some of the $G$-band brightenings, which are only visible as lengthy worm-like features in the original image, start to become resolved into chains of dots. They are similar to the ribbon-like structures seen by 

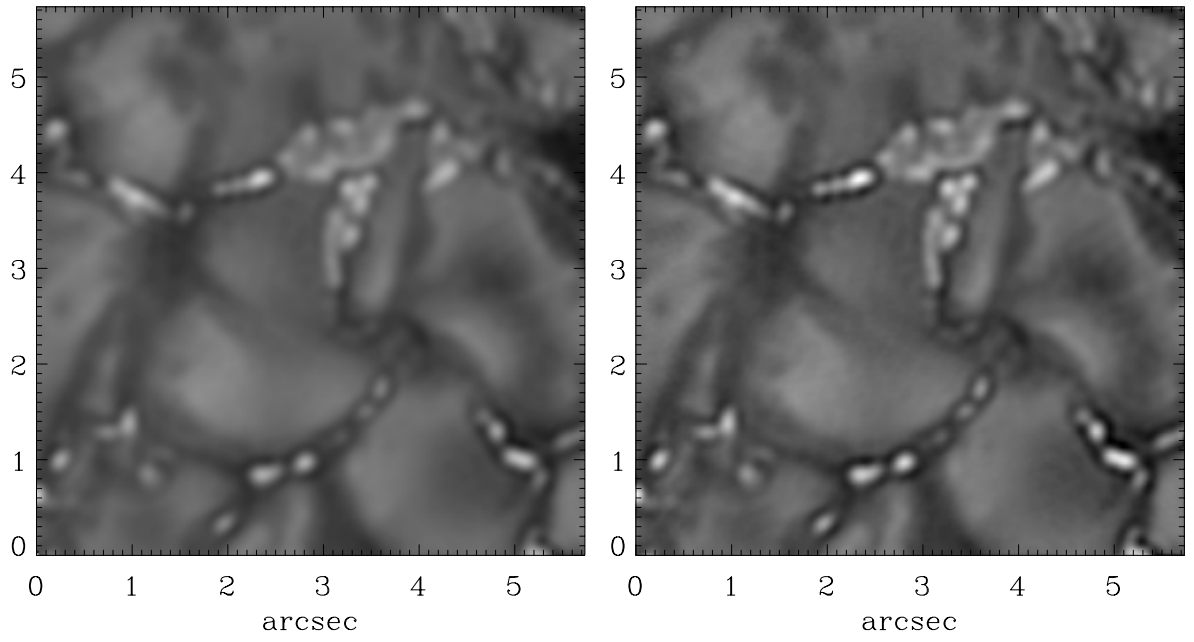

Fig. 4. Part of a $58^{\prime \prime} \times 38^{\prime \prime} G$-band image taken from an active region near disc centre in June 2004 with the $1 \mathrm{~m}$ Swedish Solar Telescope at the Observatorio del Roque de los Muchachos on La Palma. Left: original, speckle-reconstructed image; right: left image after contrast enhancement.

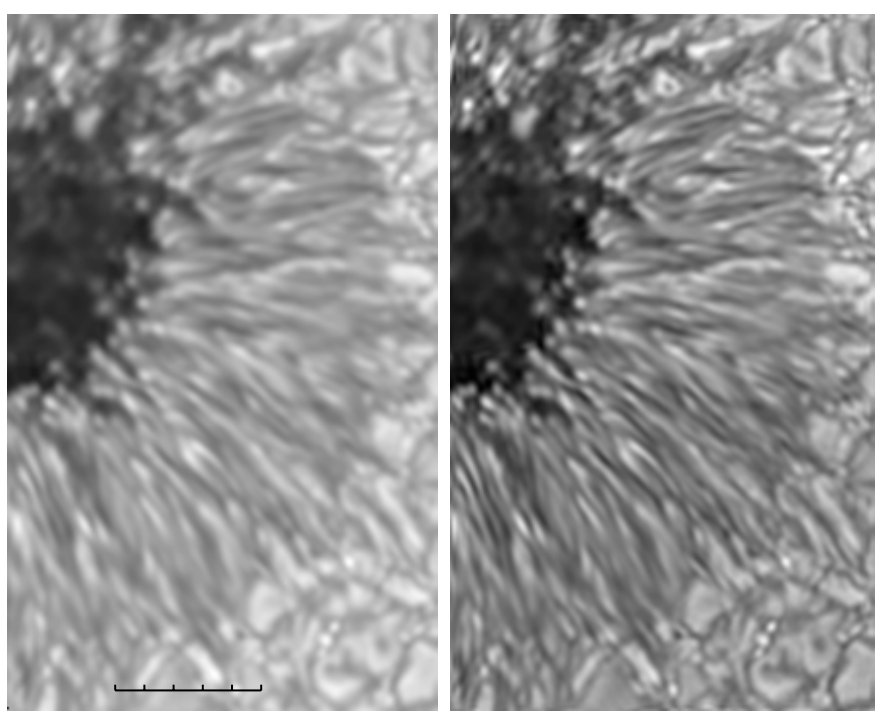

Fig. 5. Left: broadband image (after speckle reconstruction) of part of a sunspot observed at $630 \mathrm{~nm}$ in April 2002 with the $0.7 \mathrm{~m}$ German Vacuum Tower Telescope (VTT) at the Observatorio del Teide on Tenerife (from Bello González et al. 2005); right: left image after further deconvolution. The distance of the ticks in the left image is $1^{\prime \prime}$.

Berger et al. (2004, Fig. 6 in their work) which, according to these authors, are not resolvable into a series of bright points.

The next figure (5) shows the application of sharpening to a speckle-reconstructed image of a sunspot taken at $630 \mathrm{~nm}$ with the German Vacuum Tower Telescope (VTT) on Tenerife. It stems from a data set analysed by Bello González et al. (2005) and contains the centre side penumbra of the sunspot. The pixel distance corresponds to 0 .' 105 , which means a slight undersampling with respect to the diffraction limit of 0 .' $^{\prime} 186$. The image was again interpolated to have three pixels across the former, originally two, pixels. The sharpening procedure was then applied. The gain in sharpness is substantial and the deconvolved image is more brilliant than the original one, presumably because the speckle reconstruction had used a conservative noise filter. The image starts to exhibit dark cores between parallel bright filaments which were detected by Scharmer et al. (2002).

\section{Conclusions}

We have shown that super-resolution, i.e. resolution beyond the telescopic diffraction limit, is possible for point-like objects like stars in agreement with Lucy (1992b, and other work in the references) and Magain et al. (1998). However, superresolution cannot be achieved for extended objects which are not band-limited.

Point sources, on the one hand, can be represented as Dirac $\delta$-functions that possess signatures at all wavenumbers in the Fourier domain. Thus, as is shown in Eqs. (4) and (5) above, the information about photometric intensity and position is also available from low angular wavenumbers. Very high angular resolution with high photometric precision is possible, even with small telescopes, if it includes the strong a priori knowledge that one deals with point sources in the field of view. The underlying data must have a high signal to noise ratio, though. In our simplified example we reconstruct the image of two stars of equal brightness with angular separation a factor 2.5 smaller than Rayleigh's resolution limit. We found it convenient to work in the Fourier domain because then the derivatives of the error metric with respect to the quantities being sought are easily written down.

On the other hand, extended objects without bandlimitation, such as the granulation of the Sun, possess small-scale fluctuations, i.e. at high wavenumbers. Then the modulation transfer functions of the imaging processes act as low-pass filters, and information at angular wavenumbers beyond the cutoff cannot be retrieved any more. For instance the granular intensity pattern in Fig. 2 above derived from a numerical simulation of granular convection by the Lindau-Chicago group (Vögler et al. 2005), here considered as "ground truth", exhibits many edges, ridges, and spines. It appears impossible to guess their length, position, and orientation a priori and to use this prior information for reconstruction beyond the diffraction limit.

We finally applied a reconstruction that acts as a Wiener filter to two observed speckle-reconstructed images of the Sun. It is seen in both examples, a $G$-band image from an active region and a broadband image at $630 \mathrm{~nm}$ from a sunspot, that sharpening, in the sense of increasing contrast but not angular 
resolution beyond the diffraction limit, gives valuable information for studies of solar finestructure. For higher angular resolution, however, telescopes with larger apertures are needed.

Acknowledgements. K.G.P. thanks the Deutsche Forschungsgemeinschaft for support through grant KN 152/29-1. The Vacuum Tower Telescope is operated by the Kiepenheuer-Institut für Sonnenphysik, Freiburg, at the Spanish Observatorio del Teide on Tenerife and the Swedish Solar Tower by the Institute for Solar Physics of the Royal Swedish Academy of Sciences at the Observatorio Roque de los Muchachos on La Palma of the Instituto de Astrofísica de Canarias.

\section{References}

Bello González, N., Okunev, O. V., Domínguez Cerdeña, I., Kneer, F., \& Puschmann, K. G. 2005, A\&A, 434, 317

Berger, T. E., Rouppe van der Voort, L. H. M., Löfdahl, M. G., et al. 2004, A\&A, 428, 613

Biraud, Y. 1969, A\&A, 1, 124

Born, M., \& Wolf, E. 1970, Principles of Optics, 4th ed. (Pergamon Press)

de Boer, C. R. 1996, A\&AS, 120, 195

de Boer, C. R., \& Kneer, F. 1992, A\&A, 264, L24
Hook, R. N., \& Lucy, L. B. 1994, in The Restoration of HST Images and Spectra II, ed. R. J. Hanisch, \& R. L. White (Baltimore: Space Science Institute), 86

Lucy, L. B. 1974, AJ, 79, 745

Lucy, L. B. 1992a, A\&A, 261, 706

Lucy, L. B. 1992b, AJ, 104, 1260

Lucy, L. B. 1994a, Astronomical Inverse Problems, Rev. Mod. Astron., 7, 31

Lucy, L. B. 1994b, A\&A, 289, 983

Lucy, L. B. 1994c, in The Restoration of HST Images and Spectra II, ed. R. J. Hanisch, \& R. L. White (Baltimore: Space Science Institute), 79

Lucy, L. B., \& Walsh, J. R. 2003, AJ, 125, 2266

Magain, P., Courbin, F., \& Sohy, S. 1998, A\&A, 494, 472

Miura, N., Baba, N., Sakurai, T., et al. 1999, Sol. Phys., 187, 347

Richardson, W. H. 1972, J. Opt. Soc. Am., 62, 55

Scharmer, G. B., Gudiksen, B. V., Kiselman, D., Löfdahl, M. G., \& Rouppe van der Voort, L. H. M. 2002, Nature, 420, 151

Shelyag, S., Schüssler, M., Solanki, S. K., Berdyugina, S. V., \& Vögler, A. 2004, A\&A, 427, 335

von der Lühe, O. 1984, J. Opt. Soc. Am. A1, 510

Vögler, A., \& Schüssler, M. 2003, Astron. Notes/AN, 324, 399

Vögler, A., Shelyag, S., Schüssler, M., et al. 2005, A\&A, 429, 335

Weigelt, G. P. 1977, Opt. Comm., 21, 55

Wittmann, A. 1971, Sol. Phys., 21, 237 